\title{
Intermédialités
}

Histoire et théorie des arts, des lettres et des techniques

Intermediality

History and Theory of the Arts, Literature and Technologies

\section{Le tango argentin entre apprentissage et improvisation. Quel média pour quel reenactment?}

\section{Valeria De Luca}

Numéro 28-29, automne 2016, printemps 2017

refaire

redoing

URI : https://id.erudit.org/iderudit/1041085ar

DOI : https://doi.org/10.7202/1041085ar

Aller au sommaire du numéro

Éditeur(s)

Revue intermédialités (Presses de l’Université de Montréal)

ISSN

1920-3136 (numérique)

Découvrir la revue

Citer cet article

De Luca, V. (2016). Le tango argentin entre apprentissage et improvisation. Quel média pour quel reenactment ? Intermédialités / Intermediality, (28-29). https://doi.org/10.7202/1041085ar
Résumé de l'article

Inscrit en 2009 sur la liste du patrimoine culturel immatériel de l'humanité de I'UNESCO, le tango argentin est transmis par sa propre pratique caractérisée par l'improvisation de la milonga (le bal).

Avec la multiplication de traces audiovisuelles diverses - des vidéos de démonstration aux systèmes de motion capture, en passant par des sites web et des applications pour l'apprentissage de la danse - il s'agit de comprendre les relations entre l'improvisation comme pratique fondatrice du tango et ses diffractions médiales, ainsi que le statut de ces diffractions. Quels aspects du mouvement et de la pratique restituent-elles ? Sont-elles elles-mêmes un reenactement médial se superposant à celui des corps des sujets ? 


\title{
Le tango argentin entre apprentissage et improvisation. Quel média pour quel reenactment?
}

\author{
Valeria De luca
}

e tango argentin, en tant que phénomène culturel complexe mêlant plusieurs productions sémiotiques - de l'interaction corporelle à la constitution d'imaginaires identitaires -, exemplifie certains questionnements concernant la notion de reenactement ainsi que certaines problématiques liées aux médias par lesquels le tango peut être transposé.

Inscrit en 2009 sur la liste du patrimoine culturel immatériel de l'humanité de l'UNESCO, le tango voit assurée sa transmission grâce au dispositif d'improvisation qui gère sa constitution en tant que danse et grâce aux pratiques moyennant lesquelles le mouvement existe concrètement. Si d'un côté la composition musicale s'est partiellement affranchie de l'oralité, d'un autre côté la danse témoigne d'une résistance envers certaines formes d'écriture. Malgré la présence de quelques tentatives de transcription du mouvement, c'est par l'activité et la mémoire corporelles que des figures et des styles de danse se sont stabilisés, et ce, en dépit de leur fragilité constitutive. La multiplication actuelle de traces audiovisuelles diverses - des vidéos de démonstration aux systèmes de motion capture en passant par des sites web et des applications pour l'apprentissage de la danse - ajoute des couches à la fois de médiation et de médiatisation qui complexifient aussi bien l'interaction corporelle improvisée du tango que l'organisation de ses pratiques (dont notamment la pratique d'apprentissage). Dès lors, il s'agit d'examiner les relations entre l'improvisation comme pratique fondatrice du tango et ses diffractions médiales. On essayera de comprendre quels aspects du mouvement et de la pratique sont 
éventuellement restitués par ces diffractions médiales. En particulier, on se demandera si on peut les considérer comme des formes de reenactement médial à part entière et par là même, si elles peuvent se superposer au reenactement des corps des sujets.

Le choix de se concentrer tout particulièrement sur les formes de « diffraction » médiale du reenactement du geste dansant du tango est motivé par différentes raisons, à la fois épistémologiques, théoriques et méthodologiques. D’un point de vue épistémologique, comme nous l'avons montré ailleurs ${ }^{1}$, les recherches en sciences humaines et sociales - sociologie, ethnomusicologie, anthropologie, etc. - autour du tango - ont porté, dans la quasi-totalité des sources considérées, sur : i) la genèse, l'histoire et l'évolution du tango, notamment du point de vue musical (genèse et évolution de la musique, rôle des instruments musicaux, etc.) ainsi que socioculturel (par exemple : histoire et évolution du tango au sein de différents groupes sociaux, thématisation des rôles de «genre » dans les chansons et dans la pratique du tango); ii) l'histoire et les « politiques » corporelles du tango en tant que danse, comme dans le cas des débats anciens sur ses inconvénients, ou de ceux actuels sur ses bienfaits en termes médicaux, corporels, psychiques et expérientiels, ou encore, dans le cas de l'identification des traits originaires du tango, de la classification et l'explication de sa structure, de ses pas et figures; iii) la langue et les paroles du tango dans la double acception du mot, telles qu'en témoignent les recherches sur l'origine du mot «tango », sur le lunfardo, à savoir le type de sociolecte/pidgin spécifique parlé par les premiers immigrés et habitants de la ville de Buenos Aires et devenu langue des paroles des tangos, ainsi que les analyses sur les mêmes chansons. Cela a dessiné un tableau (inter)disciplinaire dans lequel le phénomène tango se présente comme quelque peu morcelé par des coupures effectuées en fonction de ses supports de manifestation, à savoir le corps pour la danse, la chanson pour la musique, la langue pour la littérature et l'identité culturelle au sens large du terme. En revanche, notre propre approche théorique et méthodologique, qui s'inscrit dans une perspective sémiotique d'inspiration post-structuraliste et phénoménologique, vise à interroger et à décrire le tango en sa globalité en tant que phénomène multidimensionnel, c'està-dire en essayant d'articuler entre elles ses différentes formes de manifestation à partir d'une focalisation sur le lien étroit entre l'interaction corporelle de la danse et la pratique du bal. L'adoption d'une pareille perspective implique d'un côté un

${ }^{1}$ Nous nous permettons de renvoyer à Valeria De Luca, Les univers sémiotiques de la danse. Formes et parcours $d u$ sens dans le tango argentin, thèse de doctorat, Université de Limoges, 2016, disponible en ligne : https://tel.archives-ouvertes.fr/tel-oI466263/document. 
changement du regard porté sur l'échange kinesthésique de l'interaction dansante en tant que tel et, de l'autre côté, une réflexion et un réaménagement des modes d'articulation entre les différentes dimensions à la base de l'émergence et de la stabilisation des valeurs et, globalement, du «sens » du tango. Par conséquent, au lieu d'examiner par exemple les contraintes musculaires, mécaniques, toniques de l'interaction et de l'improvisation dansante — comme en témoignent maintes études aussi bien en danse que plus précisément sur le tango ${ }^{2}-$, il s'agit pour nous de concevoir le tango en tant que forme sémiotique à part entière. Dans une ligne qui va de l'acception gestaltiste du terme à ses reprises en sémiotique et dans les recherches sur la morphogenèse ${ }^{3}$, toute forme, qui est à la fois perceptive et culturelle, émerge et se stabilise à partir d'un fond, sans pour autant s'en détacher complètement: ses variations, tout comme ses retours partiels vers le fond, sont en effet dans un rapport qui est de l'ordre de la motivation et de l'expression ou, autrement dit, elles dépassent la mère configuration de ses parties pour relever en revanche d'une relation chiasmatique entre assomption subjective et capacité structurante des attestations et des productions culturelles. De ce fait, l'attention se déplace vers la transposition et la reprise de toute forme dans des dimensions, des plans ou des niveaux autres de la constitution du sens. Dans ce cadre, l'interrogation sur le reenactement et sur les formes de médiatisation du tango répond à une exigence méthodologique plus ample, à savoir l'exploration des passages entre interaction culturelle et pratique ${ }^{4}$, avec cette fois, l'implémentation d'une couche supplémentaire représentée précisément par l'environnement technique et les différents outils technologiques qui seront décrits dans les lignes suivantes. À cet égard, il faut également préciser qu'un examen sémiotique de ce type de forme de médiatisation par rapport au tango est tout à fait inédit et récent car cette discipline s'est pendant longtemps écartée de l'étude de la danse tout court, et ce, malgré la présence de plusieurs approches et de tentatives de modélisation. Dès lors, l'étude que nous présentons ici a un caractère exploratoire, visant notamment à fournir des premières distinctions entre différents outils et

2 Voir Marito Olsson-Forsberg, «Construire l'état de danse à travers l'empathie kinesthésique. L'exemple de l'apprentissage du tango argentin », Staps, vol. IO2, $\mathrm{n}^{\circ} 4$, 2013, p. 88-89, http://www.cairn.info/revue-staps-2013-4-page-89.htm (consultation le 8 mai 2017).

3 Voir à ce propos, entre autres, René Thom, Apologie du logos, Paris, Hachette, 1990; et Pierre Cadiot et Yves-Marie Visetti, Pour une théorie des formes sémantiques. Motifs, profils, thèmes, Paris, Presses universitaires de France, 20or.

4 Voir Jacques Fontanille, Sémiotique des pratiques, Paris, Presses universitaires de France, 2008. 
supports de médiation et des premières suggestions sur la portée spécifiquement sémiotique de la notion de reenactement.

\section{LE REENACTEMENT ET LE TANGO}

Les suggestions qui suivent s'appuient sur une hypothèse de fond, suivant laquelle le tango peut être lui-même considéré comme une forme et une pratique de reenactement dans une acception large du terme. En effet, le tango semble exemplifier deux volets principaux de pertinence et d'application de la notion de reenactement, à savoir, d'un côté, la remémoration performative d'événements historiques et, de l'autre côté, l'acte performatif en tant que tel des performances artistiques (musicales, théâtrales, dansantes). En particulier, la notion de reenactement permet de montrer premièrement l'épaisseur temporelle de tout acte ou geste - une épaisseur qui est à la fois culturelle, politique et esthétique. Deuxièmement, elle permet par conséquent de voir sous un nouveau jour la nature même de ce qui est reenacté. Plus précisément, en exhibant le caractère toujours de médiation des actes, des gestes, des événements — « médiés » par des facteurs ou des contraintes de type politique, technique, social, etc. - la notion de reenactement permet de tenir ensemble deux aspects que la répétition de gestes et de pratiques sociales et culturelles implique. Ces deux aspects sont: i) une dramaturgie normative propre à toute pratique (distribution et assomption de rôles, microrituels internes aux pratiques qui en scandent son propre rythme, tactiques de régulation des conflits éventuels, etc.), et ii) la mise en exergue du potentiel de variation et d'indétermination activé par la répétition même ou, en d'autres termes, ses propres «produits ». Ces produits se manifestent sous des formes diverses : des traces, mais également des résidus, des déchets, des survivances ${ }^{5}$, tout ce qui excède les cadres normatifs et qui peut en même temps être « récupéré » par une forme de vie lorsqu'elle revient réflexivement sur elle-même à travers l'instauration de nouvelles formes de médiation. Tout cela est manifesté par les différentes conceptions et définitions du reenactement. Parmi les nombreuses contributions existantes, nous retenons notamment les suggestions que l'on repère chez Vanessa Agnew, André Lepecki et Rebecca Schneider. Vanessa Agnew mentionne d'abord le caractère émancipateur du geste de reenactment qui permettrait que les sujets impliqués puissent « to select their own past in reaction to

5 Voir la notion d'《image survivante » proposée par Georges Didi-Huberman dans L'image survivante : histoire de l'art et temps des fantômes selon Aby Warburg, Paris, Les Éditions de Minuit, coll. « Paradoxe », 20II. 
a conflicted present ${ }^{6} \gg$; en même temps, cette émancipation et cet engagement sont rendus possibles par l'a-bistoricité constitutive de tout reenactement et qui est relié à la part de fictionnalisation qu'il comporte. Néanmoins,

This historical fallacy does not, perhaps, detract from its heuristic usefulness. It opens up the past as a realm of foreclosed possibilities and interrogates the specific conditions of those foreclosures. The object is not a historical account of the past "as it reallay was" but an opening to more fruitful interpretations. [...] Rather than eclipsing the past with its own theatricality, reenactement ought to make visible the ways in which events were imbued with meanings and investigate whose interests were served by those meanings ${ }^{7}$.

En réalité, la fictionnalisation introduite par le reenactement, plutôt que signaler l'a-bistoricité de celui-ci, fait affleurer - nous semble-t-il - la pluriactantialité de l'histoire même, du moins si l'on suit la lecture qu'Emmanuel Bouju fait de la distinction benvenistienne entre l'bistor (-juge) et l'istor (-témoin) par rapport à l'(h)istoricisation en littérature, où la fiction du témoin occupe à bon droit «cette place interstitielle, entre deux garants (entre le témoin oculaire et le juge, l'arbitre) » en faisant de leurs énonciations «le moyen [...] d'une "autopsie" (une vue par soi-même), au présent, du passé $^{8} \gg$. Ce sont précisément, d'une part, la brisure de la linéarité temporelle et, d'autre part, l'hétérogénéité et la multimodalité des gestes et des « faits » reenactés qui motivent la conception « résiduelle d de la performance et du reenactement chez Rebecca Schneider. Sous sa plume, le reenactement trouble la linéarité temporelle « by offering at least the suggestion of recurrence, or return, even if the practice is peppered with its own ongoing incompletion $^{9} \gg$. De ce fait,

If the past is never over, or never completed, "remains” might be understood not solely as object or document material, but also as the immaterial labor of bodies engaged in and with that incomplete past: bodies striking poses, making gestures, voicing calls, reading words, singing songs $[\ldots]$. To find the past resident in remains-material evidence, haunting trace, reiterative

${ }^{6}$ Vanessa Agnew, «Introduction: What is Reenactment? », Criticism, vol. 46, ${ }^{\circ} 3$, été 2004, p. 328.

7 Ibid., p. 334-335.

${ }^{8}$ Emmanuel Bouju, « Note sur l'istoricisation de la fiction », Acta fabula, vol. I4, n5 «L'aire du témoin », juin-juillet 2013, www.fabula.org/revue/document7988.php (consultation le 8 mai 20I7).

9 Rebecca Schneider, Performing Remains: Art and War in Times of Theatrical Reenactment, Londres, Routledge, 2oII, p. 30. 
gesture - is to engage one time resident in another time-a logic rooted in the word "remain." Time, engaged in time, is always a matter of crossing, or passing, or touching, and perhaps always (at least) double ${ }^{10}$.

Le propre du reenactement est dès lors représenté par l'incorporation et par la monstration dans les gestes et les pratiques de ces « résidus » mêmes qui, d'une part, permettent à une forme de vie de se renouveler et de persister dans le temps et, d'autre part, constituent les candidats pour l'impulsion à l'archive propre à toute culture et, par conséquent, pour tout geste instituant une tradition et un patrimoine sur la base d'une authenticité fabriquée $e^{11}$ (même lorsqu'elle est revendiquée comme telle).

Le caractère pour ainsi dire «fictionnel » de l'archive et le vecteur de déterritorialisation que les matériaux résiduels introduisent dans la circulation des formes dansantes - telles que les figures et les styles de danse du tango - sont exemplifiés par André Lepecki lorsqu'il reconduit l'«impulsion à l'archive » à la capacité « to identify in a past work still non-exhausted creative fields of "impalpable possibilities"12 $\gg$.

Dans cette perspective, l'interaction dansante improvisée du tango, effectuée dans un cadre pratique qui est émergé lui-même de manière improvisée - comme nous avons essayé de le montrer ailleurs ${ }^{13}$ - , constitue dès le départ une forme de reenactment par rapport d'une part aux inscriptions fictionnelles par exemple des paroles des chansons et, d'autre part, par rapport aux normes pratiques mêmes (celles de la pratique du bal) qui, en revanche, ne sont légitimées qu'une fois reprises par d'autres pratiques (les championnats de tango).

\section{COORDONNÉES D'UNE ÉMERGENCE IMPROVISÉE}

La persistance du tango argentin au fil du temps (et par là même sa transmission) est garantie précisément par l'improvisation comme modalité de l'effectuation pratique du tango en ses plusieurs déclinaisons. Il s'agit d'une modalité

Io Ibid., p. 33 et 37 .

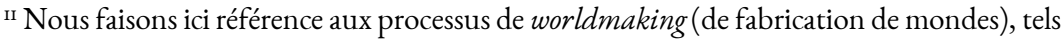
que décrits par Nelson Goodman dans son Manières de faire des mondes, Nîmes, Jacqueline Chambon, 1992. Pour une contextualisation de la notion de worldmaking en danse, voir Gabriele Klein et Sandra Noeth (dir.), Emerging Bodies. The performance of Worldmaking in Dance and Choreography, Bielefeld, Transcript Verlag, 20II. Nous nous permettons de renvoyer aussi à De Luca, 2016.

${ }^{12}$ André Lepecki, « The Body as Archive: Will to Re-Enact and the Afterlives of Dances $\gg$, Dance Research Journal, vol. $42, \mathrm{n}^{\circ} 2$, hiver 20Io, p. 3I.

${ }^{13}$ Voir De Luca, 2016. 
qui concerne l'interaction corporelle dansante dans le cadre d'une pratique spécifique - la milonga, le bal - et en mesure mineure, la composition musicale. De surcroît, il faut rappeler que le tango « rioplatense », adjectif qui fait référence à la zone appelée Río de la Plata marquant la frontière entre Argentine et Uruguay, a été classé par l'UNESCO en 2009 patrimoine culturel immatériel de l'Humanité, dont la définition donnée par l'UNESCO est la suivante :

Le Tango est un genre musical qui associe danse, musique, poésie et chant, et qui est considéré comme l'une des principales manifestations de l'identité des habitants de la région de Río de la Plata. L'inscription de l'élément sur la Liste représentative contribuerait à la visibilité du patrimoine culturel immatériel et à une connaissance plus approfondie du Tango en tant qu'expression régionale résultant de la fusion de plusieurs cultures ${ }^{14}$.

À ce propos, le projet « Patrimonio vivo » durant l'année 2013 a organisé une série d'ateliers avec l'objectif de circonscrire un premier périmètre d'observation et d'esquisser un inventaire des formes vivantes du tango. Malgré l'intérêt de ces nombreuses initiatives, les problèmes qui se posent à l'égard de la « patrimonialisation » de la danse et plus particulièrement de l'interaction dansante de la milonga, du bal, demeurent. Comme l'a remarqué l'historienne Liliana Barela ${ }^{15}$, les questionnements concernent les manières de sauvegarder le présent chaque fois différent qui constitue la spécificité de l'improvisation dans le tango. Par conséquent, il s'agit de comprendre comment tenir compte à la fois des aspects plus stabilisés de la pratique du tango et de la singularité de l'acte de danse. Cette singularité n'est pas qu'individuelle mais est ancrée dans le devenir même du tango. Elle relève notamment des diatribes concernant les styles de danse et des revendications autour de l'authenticité de chacun d'eux qui se prolongent jusqu'à présent sur deux fronts, l'un géographiquement «intérieur », qui tient aux catégories de styles de tango officialisées dans les deux Championnats de tango qui ont lieu chaque année à Buenos Aires, et l'autre front, « extérieur », qui concerne les déclinaisons du tango dans le monde entier, ce qui a été appelé le phénomène de trans-régionalisation ou trans-

I4 Voir UNESCO, «Décision du comité intergouvernemental: 4.COM I3.OI », Patrimoine culturel immatériel, 2009, www.unesco.org/culture/ich/fr/decisions/4.COM $/ 1$ 3. or ?dec=decisions\&ref_decision=4.COM (consultation le 8 mai 20I7).

is Liliana Barela a été directrice générale du patrimoine et des institutions historiques de la ville de Buenos Aires. Elle a suivi depuis les années 2000 la démarche de candidature du tango en tant que patrimoine auprès de l'UNESCO. 
culturation $^{16}$. Du coup, quels sont les dimensions et les niveaux qui permettent de dégager les spécificités identitaires du tango à partir d'un cadre de variation continue, à la fois historique, corporelle, sociale et imaginaire ? Comment peuvent-elles rendre compte de la construction identitaire « rioplatense » sans aplatir la charge culturelle du tango sur des critères étatiques ou géographiques? Les experts de l'UNESCO se sont eux-mêmes engagés dans cette direction en reformulant partiellement en $201 \mathrm{l}$ la déclaration relative au tango et ont étendu le patrimoine culturel immatériel à ce qui est « traditionnel, contemporain et vivant en même temps » et en remplaçant l'idée d'authenticité par celle de « continuité historique » qui admet des déplacements, des adaptations et des récréations.

\section{L'IMPROVISATION ET LA PRATIQUE}

Dans le tango, on peut parler d'improvisation en ceci qu'un seuil technique minimum permet et co-existe avec la création d'une forme inédite dans le cours même de l'action, sans que ce soit possible d'en prédire la suite et dans un cadre normatif instable. En particulier, un ensemble de figures, d'enchaînements de mouvements ou des gestes, qui ne s'identifient pas avec des pas de danse, comme dans le cas du ballet classique, s'affirment au fil du temps et acquièrent des appellations telles que le ocho (huit), la salida (littéralement la sortie), le gancho, la volcada, etc.

La maîtrise des figures, ainsi que leur enchaînement, ne dérive pas d'un ordonnancement préalable où une figure en engendre une autre, et ainsi de suite. $\mathrm{Au}$ contraire, l'incorporation et l'effectuation des figures résultent uniquement de la maîtrise de variations tensives/rythmiques opérées sur la forme gestuelle et sur ses composantes (posture, balancement du poids, système de marquage, autrement dit marcación) et, par conséquent, de combinaisons entre les mouvements du haut du corps et ceux du bas. Ce ne sont pas les éléments pris singulièrement qui permettent de différencier une figure d'une autre, mais leurs variations, car une variation sur le rythme (une pause ou bien une accélération) peut permettre par exemple d'insérer un gancho ou une parada à l'intérieur d'un tour. Il y a là une première déclinaison de l'improvisation, concernant l'apprentissage des figures. À ce sujet, il faut préciser que, depuis ses débuts et jusqu'aux années 1950 et au début des années 1960, le tango tel qu'il était dansé au moins à Buenos Aires ne se fondait que sur la pratique du bal, de la milonga. Autrement dit, il n'y avait pas de séparation spatiale ou temporelle entre

${ }^{16}$ Voir Ramón Adolfo Pelinski (dir.), El tango nómade. Ensayos sobre la diáspora del tango, Buenos Aires, Corregidor, 2000. 
la pratique d'apprentissage et celle de la danse en tant que telle. Le savoir-faire de la danse reposait sur une base fiduciaire en fonction non seulement de la compétence technique des milongueros (les adeptes reconnus, de véritables personnages de la milonga), plutôt que sur leur adhérence à la pratique, sanctionnée par l'évaluation des conduites sur tous les plans : des tenues vestimentaires à l'occupation normée de l'espace, en passant par les variations gestuelles admises et interdites. En somme, le savoir-faire était dépendant d'un savoir-être qui privilégiait la capacité des acteurs à assumer le cours de l'action, à faire de l'improvisation l'emblème d'une norme naissante, dans la mesure où, en dehors des moments d'apprentissage partagés entre danseur novice et danseur confirmé, le reste était confié à l'observation et à la transmission orale.

$I_{13}$ Dès lors, l'improvisation proprement dite, celle qui s'effectue dans l'interaction dansante, semble dès le départ ancrée dans les deux pratiques à la fois, celle de l'apprentissage et celle du bal. L'improvisation dansante double à cause de deux constats : i) le bal et les morceaux de musique sont scandés d'une manière (l'alternance entre tandas, des séquences de trois ou quatre morceaux, et cortinas, un morceau autre que le tango) telle que l'on change de partenaire idéalement à chaque tanda et ii) par conséquent, le fait de se retrouver constamment face à un corps inconnu, avec lequel devoir s'ajuster. Dans l'interaction dansante, l'improvisation concerne également le mode dans lequel l'acte de danse est poursuivi en tenant compte de l'espace global de la salle et des autres corps en mouvement. Dans les cadres de la philosophie de la danse, de nombreux auteurs se sont interrogés sur le statut de cette notion. Sans pouvoir prétendre restituer la totalité des lectures et des conceptions sur l'improvisation en danse, esquissons une ligne interprétative qui nous permettra de restituer les relations entre norme et improvisation. En premier lieu, le chorégraphe et chercheur en improvisation Jacques Gaillard avance qu' « improviser suppose qu'on s'inscrive dans une visée de laquelle l'horizon est absent. [...] J'aimerais [...] montrer que l'improvisation est tout sauf un abandon, qu'elle nécessite une structure, implique finalement la conscience, le jeu de l'attention et, paradoxe, un contrôle que faute de mieux, je qualifierai aujourd'hui d'émergent ${ }^{17}$. » La visée qui s'inscrit est celle qui, par une exploration perceptive, est en mesure de figurer un tel horizon, c'est-à-dire de donner consistance à un paysage

${ }^{17}$ Jacques Gaillard, « L'improvisation dansée : risquer le vide. Pour une approche psychophénoménologique », dans Anne Boissière et Catherine Kintzler(dir.), Approche philosophique du geste dansé. De l'improvisation à la performance, Villeneuve d'Ascq, Presses universitaires du Septentrion, coll. « Esthétique et sciences des arts », 2006, p. 7I. 
de formes disponibles susceptibles d'une détermination. En mettant l'accent sur les actes attentionnels par rapport à l'intention, il souligne que le noyau de l'improvisation réside dans le risque à l'imprévu, contrairement au motif du vide qui domine plusieurs lectures de l'improvisation. Cet imprévu, ainsi que la charge d'attention qu'il comporte, relève dès lors d'un processus d'évaluation, d'anticipation d'un scénario à la fois possible et nécessaire que les premiers gestes dégagés permettent d'engendrer, compte tenu des contraintes physiques et des réponses des autres acteurs impliqués. Cette perspective, située et implicitement relationnelle, permet au chorégraphe de réintroduire et de mettre en valeur la part de structuration que toute visée implique, même à un stade germinatif : il relève en effet que « le paradoxe de l'activité d'improvisation est qu'elle associe simultanément deux orientations de pensée contradictoires : une structure très forte et une liberté absolue. Sans la structure, l'improvisation se perd dans la vacuité; sans la liberté, elle perd sa visée, qui est son sens. Le nœud de l'improvisation consiste à dénouer cette énigme : qu'est-ce qu'une structure libre ${ }^{18}$ ? »

$I_{14}$ On peut constater que l'improvisation possède un caractère premier d'injonction relatif à l'attente de l'inattendu et, même, à la construction de l'inattendu. Cela implique d'une part l'invention de règles et, d'autre part, la distribution, dans un cadre partagé entre plusieurs acteurs, de la prise d'initiative à laquelle s'accommoder, afin de donner libre cours à l'improvisation, à ne pas interrompre un flux supra-individuel. En soulignant la valeur constituante de l'improvisation, on remarque notamment l'assomption et la gestion des gestes pendant le cours d'action, une assomption qui se traduit dans l'esquisse continuelle de scénarios proto-figuratifs possibles et donc par l'évaluation constante des valences à l'œuvre. Comme l'affirme Alessandro Bertinetto :

Dans l'improvisation, le projet [...] ne précède pas l'action. [...] Dans l'improvisation, l'intention se construit dans l'action, et en tant qu'action, et non pas parce que l'improvisation est [...] dépourvue d'une intention précédente qui caractériserait les actions « normales », mais parce que c'est l'intention qui se construit normalement dans l'action et comme action, à travers les modes dans lesquels l'agent responsable de l'action fait face [...] à la situation et «fait ce qu'il arrive ». [...] L'improvisation [...] manifeste clairement le caractère autonome de la normativité dans les pratiques

${ }^{18}$ Ibid., p. 73. 
humaines (la norme se transforme dans la situation de sa propre application $)^{19}$.

Dans le cas spécifique du tango, l'anthropologue Davide Sparti met en relief l'articulation aspectuelle de l'improvisation ainsi que le mouvement réflexif du tango par rapport à lui-même, qui serait à la base à la fois de son caractère toujours émergent et de sa stabilisation pratique en termes de normes corporelles et de conduite qui peuvent être confirmées ou démenties dans l'acte de danse, dans le cours d'action :

Celui qui improvise crée dans des conditions «spectaculaires », dans le double sens suivant lequel il crée en présence de spectateurs et pendant qu'il danse [...]. L'indérogeabilité et l'état d'émergence qui caractérisent l'improvisation dérivent de cette simultanéité entre invention et production. [...] Plutôt que dans les termes de l'accès à une subjectivité supposée libre, on peut saisir l'improvisation dans la capacité de rechercher [...] de nouvelles possibilités de mouvement à partir de ce qui est déjà connu [...]. En ce sens, le tango est une danse qui revient de manière réflexive sur elle-même [...] qui invente son propre présent en se [...] ré-accordant au passé. [...] Celui qui improvise est tourné vers le passé. Mais non pas jusqu'à ce que le passé (pré)détermine ce qu'il arrive maintenant, en closant le potentiel d'ouverture introduit par l'improvisation et en transformant le savoir sédimenté en un bagage de séquences à reproduire de manière procédurale ${ }^{20}$.

19 « nell'improvvisazione il progetto [...] non precede l'azione [...] nell'improvvisazione l'intenzione si costruisce nell'azione, e come azione, non perché l'improvvisazione sia [...] priva di un'intenzione precedente l'azione che caratterizzebbe le azioni "normali”, ma perché normalmente l'intenzione si costruisce nell'azione e come azione, attraverso il modo in cui l'agente responsabile dell'azione si confronta adattivamente ed esattivamente con la situazione e "fa ciò che accade" [...] l'improvvisazione [...] manifesta con particolare evidenza il carattere autonomo della normatività nelle pratiche umane (la norma si tras-forma nella situazione della sua stessa applicazione », Alessandro Bertinetto, « "Mind the Gap”. L'improvvisazione come azione intenzionale $\gg$, Itinera, $\mathrm{n}^{\circ}$ IO, 20I5, p. I79, I87-I88 (notre traduction).

${ }^{20}$ «Chi improvvisa crea in condizioni "spettacolari”, nel doppio senso che crea in presenza di spettatori, e che crea mentre balla [...] L'inderogabilità e lo stato di emergenza che contrassegnano l'improvvisazione derivano da questa simultaneità di invenzione e produzione [...] Più che nei termini dell'accesso a una soggettività primitiva supposta libera, l'improvvisazione si lascia cogliere nella capacità di ricercare nuove possibilità di movimento $\mathrm{a}$ partire da quanto già conosciuto [...] il tango è una danza che rientra riflessivamente su se stessa, che riarticola se stessa mentre si porta a compimento, che inventa il proprio presente ricordandosi il (ri-accordandosi al) passato [...] Chi improvvisa si rivolge al passato. Non però fino al punto che il passato (pre)determina quanto avviene adesso, chiudendo il potenziale di apertura introdotto dall'improvvisazione e trasformando il sapere sedimentato in un bagaglio di sequenze da riprodurre in maniera formulaica », Davide Sparti, Chapitre $3:$ «mprovvisa 


\section{LE REENACTMENT ENTRE MÉDIATION ET MÉDIATISATION}

Récapitulons. L'improvisation se caractérise comme modalité de l'émergence du tango dans la pratique, qui fait exister l'acte de danse en tant que tel. Il s'agit d'un pari sur le déroulement du cours d'action, qui prévoit l'ajustement en qualité de régime corporel gérant la relation à autrui (le partenaire de danse, les autres actants). De plus, la réitération de l'improvisation en tant que modalité pratique gérant l'interaction corporelle est génératrice de normes, en vertu de la charge mémorielle dont la routine - en tant que répétition - est investie.

Dans ce cadre, les « objets médiatiques »-logiciels, applications, expériences et environnements technologiques - qui sont ici examinés illustrent le dédoublement constitutif de l'agir du reenactment ou de la reprise entre, d'un côté, l'anticipation et la mise en variation que les conditions spécifiques de l'improvisation du tango comportent et, de l'autre côté, la normativité émergente qui innerve, dans le tango, la tension entre l'improvisation et ses cadres pratiques (l'apprentissage, le bal).

En d'autres termes, les reprises médiales de l'improvisation dansante semblent opérer sur les deux pôles de cette dynamique. En effet, si l'on se situe du point de vue du corps dansant, elles cherchent d'une part à constituer un support délégué chargé de la répétition à travers l'établissement préalable d'un répertoire des possibles. De plus, elles visent en revanche à incorporer une réflexivité seconde sur l'acte de répétition/variation gestuelle par rapport à la réflexivité des corps mêmes et de leur mémoire. Cette réflexivité s'avère proprement médiatique ou, pour mieux dire, elle est le résultat de la constitution d'un environnement médiatisé, d'une mise en relief de l'activité de la médiatisation en tant que telle, dans la mesure où elle

prévoit une tiercéité des dispositifs mobilisés qui devient prédictive d'autres médiations selon une hétérogénéité et une événementialité d'occasions de filtrage. C'est pourquoi le caractère actantiel générique d'un médium devient un environnement de redéterminations et de promotions des identités (média), au-delà de la juridiction d'origine et donc sans une cartographie préalable ou prévisible de ses réponses filtrantes ${ }^{21}$.

azione : il lato creativo del tango », Sul tango. L'improvvisazione intima, Bologne, Il Mulino, 20I5, format e-book (notre traduction).

${ }^{21}$ Pierluigi Basso Fossali, « Le poids éthéré de la médiatisation. De la matérialité diaphane du média à son investissement comme environnement », Actes du Congrès de l'Association française de sémiotique «Sens et médiation. Substances, supports, pratiques: matérialités médiatiques», juillet 20I5, http://afsemio.fr/wp-content/uploads/Sens-et-m\% $\mathrm{C}_{3}$ \%Agdiation.-P.-Basso-Fossali.pdf (consultation le 8 mai 20I7). 
Dans cette perspective, l'on peut distinguer deux macro-catégories dans lesquelles les reprises médiales peuvent s'inscrire : i) de véritables médias-supports, qui tendent à résoudre l'hétérogénéité de l'improvisation et de la répétition en tant que mise en variation, et ii) des médiatisations, des environnements médiatiques, qui répliquent l'indétermination propre à l'improvisation. À cet égard, il est intéressant de remarquer les pratiques visées par ces deux classes d'objets : dans le premier cas, il s'agit d'objets implémentés dans la pratique d'apprentissage de la danse, tandis que, dans le deuxième cas, il s'agit d'appareillages technologiques qui reconfigurent la pratique même de la danse.

Concernant les médias-supports, nous examinerons dans les lignes suivantes : a) un logiciel d'apprentissage, Bailemos Tango, élaboré à partir de la méthode d'apprentissage du danseur et chorégraphe Juan Carlos Copes ${ }^{22}$, et b) l'application pour smartphone iTango!, conçue par une équipe de musiciens, danseurs et ingénieurs ${ }^{23}$. Ensuite, par rapport aux environnements de médiatisation, nous examinerons : a) Tango Virus, un projet artistique de « contamination » d'un thème musical à partir de la rétroaction sur la musique de patterns gestuels exécutés par les danseurs en temps réel, et b) le projet Interactive Tango Milonga, un dispositif permettant le retour et la gestion en temps réel des mouvements des danseurs.

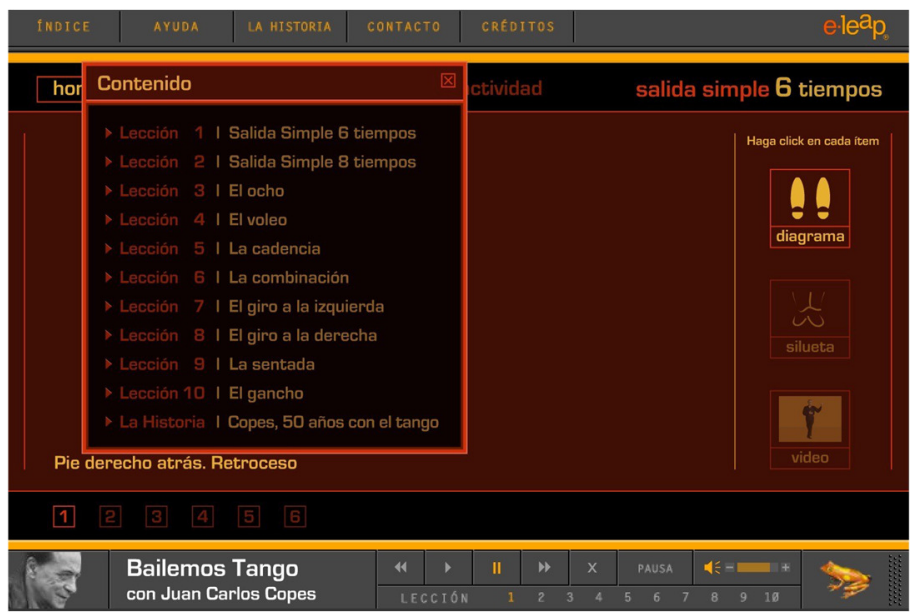

Fig. I. « Menu Contenu », capture d'écran du logiciel Bailemos Tango, Juan Carlos Copes, http://www.e-leap.com.ar/tango/sp/demo/demotango_sp.html (consultation le 8 mai 20I7).

22 Voir démo du logiciel en ligne: http://www.eleap.com.ar/tango/sp/demo/demotango_sp.html (consultation le 8 mai 20I7).

${ }^{23}$ Voir le site Internet de l'application: Magiccoding, iTango!, 2017, itango.social/ (consultation le 8 mai 20I7). 


\section{BAILEMOS TANGO}

Le logiciel Bailemos Tango résume la pédagogie élaborée au fil de quelque cinquante années de danse par le danseur et chorégraphe Juan Carlos Copes, connu notamment pour avoir intégré le corps de ballet du spectacle Tango Argentino de 1983, qui avait marqué la « renaissance » du tango à l'échelle internationale. Le logiciel peut être consulté sur internet, ou bien il peut être téléchargé en version « démo », ou, encore, il est inclus dans l'achat d'un coffret DVD. Tous les matériaux audiovisuels, enregistrements vidéo, images, instructions énoncées verbalement, sont constitués par Copes lui-même ainsi que par sa partenaire. L’objectif du logiciel étant l'apprentissage ou, à tout le moins, la révision de figures et d'enchaînements gestuels, il est organisé comme un cours virtuel et interactif, réparti en dix séances thématiques, incluant également des modules de révision guidée. La scansion séquentielle cherche à reproduire la progression présumée que l'on repère dans des cours en présentiel, bien que cette même structure s'avère moins linéaire que ce que l'on pourrait croire de prime abord. En effet, comme la figure i le montre, la séquence des cours ne relève pas que d'un ordonnancement progressif en termes de difficulté, mais aussi d'une véritable narration du parcours artistique du danseur. On assiste à un premier aspect qui nous fournit l'indice d'un reenactment hétéronome à l'égard de l'apprenti, en ceci que la séquentialité spécifique des séances affiche une trace mémorielle singularisée dont l'actuation est déléguée à l'utilisateur ${ }^{24}$. L'exécution de chaque figure est présentée de différentes manières, offrant à l'utilisateur un choix en termes de degrés d'adéquation ou de ressemblance avec l'instance productrice du reenactment hétéronome. En effet, comme on peut le voir dans l'ordre de présentation des options, on va du diagramme à la vidéo, en passant par la physionomie, qui constituent autant de déclinaisons de la constitution du geste. Plus précisément, on assiste à un passage d'une géométrisation de la figure à une incorporation de l'espace de la part des danseurs dans l'option vidéo. Les figures de 2 à 7 montrent clairement ce passage. Chaque modalité d'apprentissage et d'incorporation est appliquée aussi bien à chacun des rôles (leader et follower appelés ici plus traditionnellement homme - bombre - et femme - mujer) pris séparément qu'au couple en son unité (pareja). De surcroît, dans la section « consejos » (conseils), la voix off de Copes énonce successivement les indications fondamentales concernant la bonne forme gestuelle à adopter (voir la figure 8). Dans cette section, le recours nécessaire à l'image implique

${ }^{24}$ Il faut préciser à cet égard le caractère scénique du parcours de Copes, qui peut justifier en partie une présentation certes pédagogique, mais comprenant des figures plus spectaculaires, telles que le voleo, la sentada ou le gancho. 
une multiplication figurative de l'instance (le danseur Copes) servant de modèle. Cela crée un effet contraignant pour l'apprenti qui, bien qu'il puisse faire appel à une référence actuarielle clairement identifiée, voit son pouvoir de reconfiguration diminué par rapport à l'activité qui serait requise dans le cas de la diagrammatisation. Dès lors, d'après ces remarques, on peut affirmer que dans ce cas, bien qu'il s'agisse d'un outil d'apprentissage - et par là même de transfert de compétences -, ce média-support semble plutôt fonctionner comme une archive dans le sens opposé à l'interprétation qu'en donne André Lepecki lorsqu'il parle de « will to archive25 ». En effet, dans ce cas, ce n'est pas la répétition des figures qui est mise en valeur en tant que telle, dans sa dimension de singularisation dans la variation. Il s'agit plutôt de la reprise d'un geste à la fois singularisé - comme tout geste - et porteur d'une signature, d'une identité. Le reenactment hétéronome encouragé par ce logiciel s'avère en somme un dispositif qui permet d'un côté de constituer un répertoire gestuel et pédagogique pouvant être facilement transmis, et, d'un autre côté, un dispositif qui cherche à reproduire le processus de stylisation qui a lieu diachroniquement dans la pratique du tango, en vertu précisément de son improvisation.

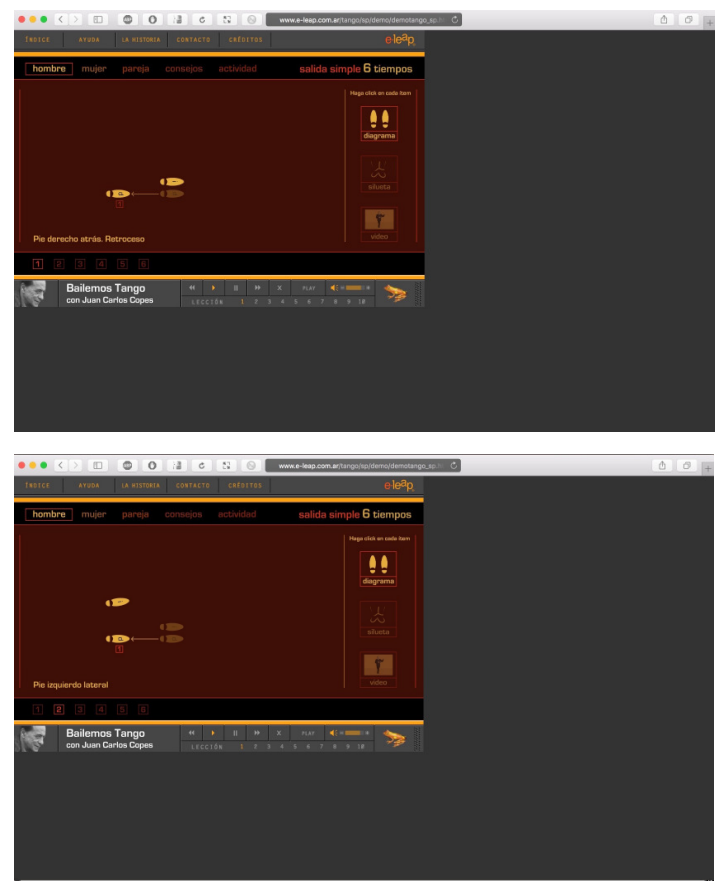

${ }^{25}$ Voir Lepecki, 2010. 

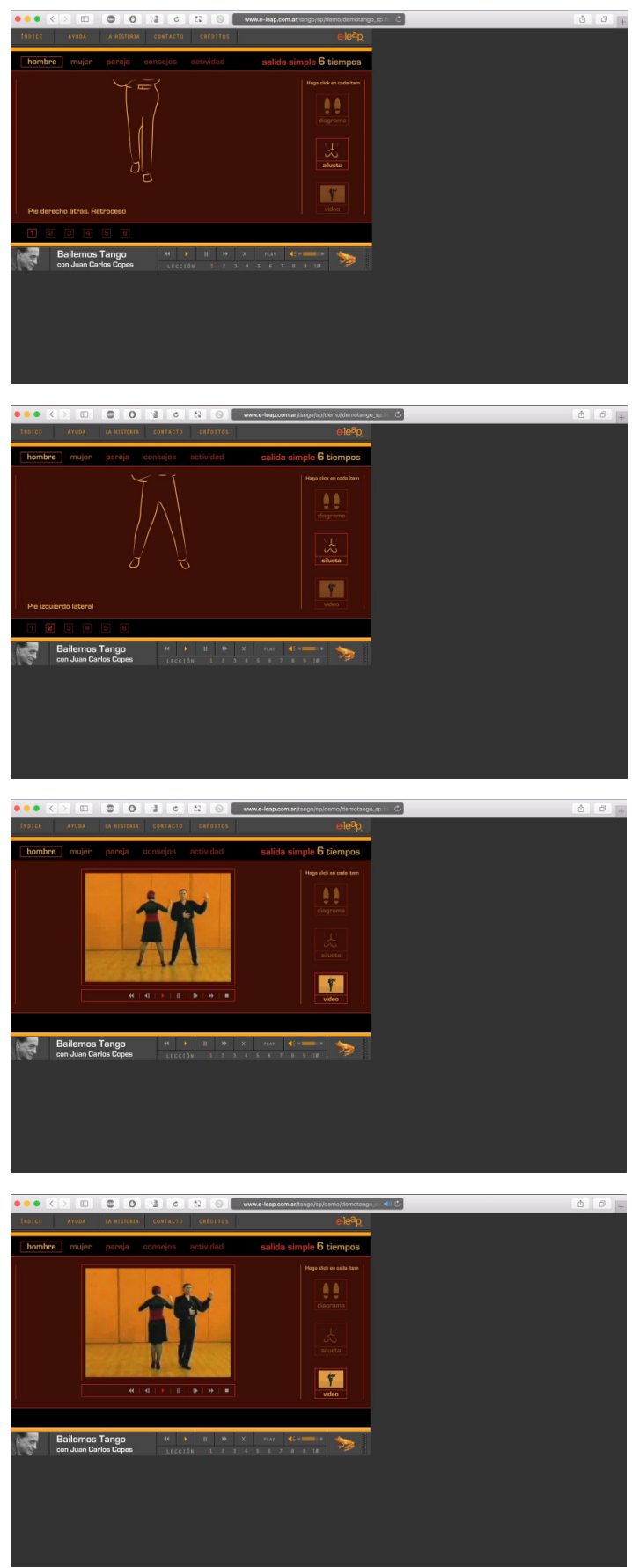

Fig. 2-7. « Explication par le diagramme I », capture d'écran du logiciel Bailemos Tango, Juan Carlos Copes, http://www.e-leap.com.ar/tango/sp/demo/demotango_sp.html (consultation le 8 mai 20I7). 


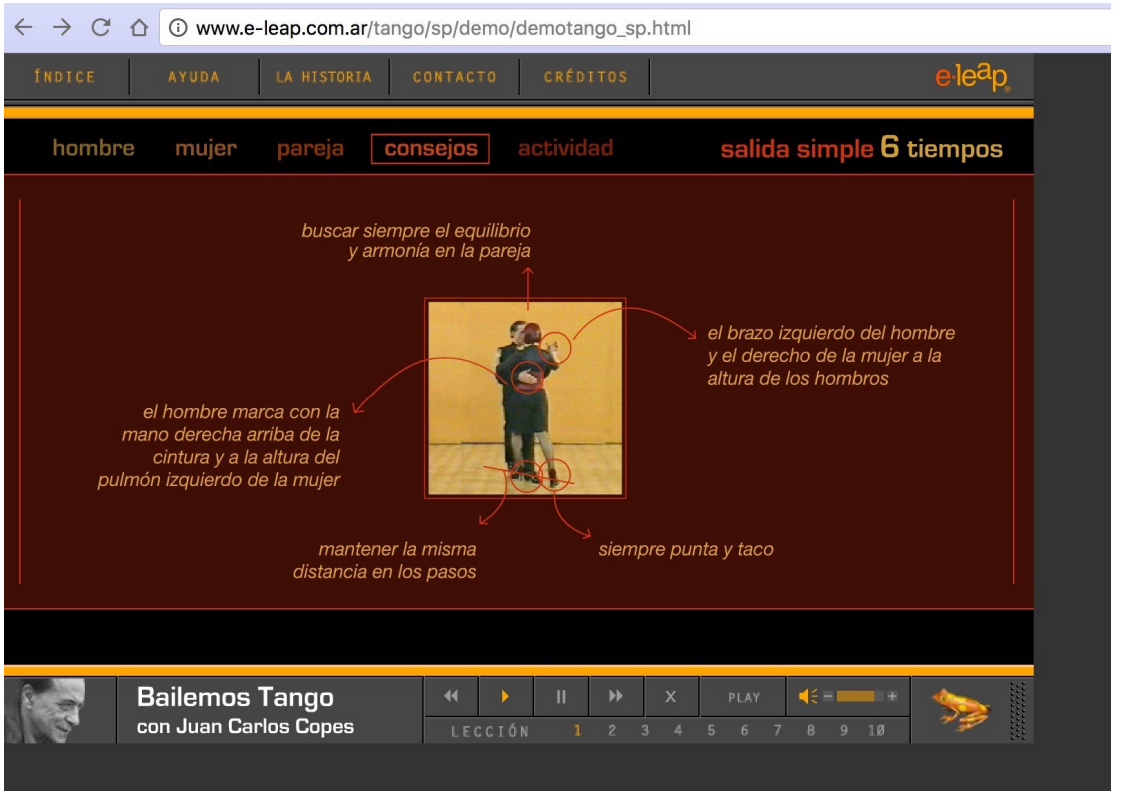

Fig. 8. « Indications posturales », capture d'écran du logiciel Bailemos Tango, Juan Carlos Copes, http://www.e-leap.com.ar/tango/sp/demo/demotango_sp.html (consultation le 8 mai 2017).

\section{ITANGO!}

Cependant, la propriété d'un média-support qui est d'être une surface d'inscription ${ }^{26}$ sur laquelle une pratique peut s'installer et intervenir, peut se décliner autrement, et ce, même dans le cas d'outils ayant des fonctions et un design relativement proches. La configuration de l'application iTango! est à cet égard emblématique, notamment si on la compare avec le logiciel précédemment mentionné. Tout d'abord, il s'agit d'une application pour téléphone intelligent, impliquant par conséquent une interaction tactile avec l'interface (voir la figure 9). En deuxième lieu, c'est la conception technique même de l'application qui, intégrée à la technologie portable du téléphone intelligent, en fait un support presque embarqué, visant à une incorporation dans et avec le corps de l'apprenti. Cela engendre à notre avis la constitution de plages pour un reenactment, pour une reprise autonome, tout en restant dans le cadre d'une pratique d'apprentissage. Examinons de plus près l'application. Parmi ses différentes caractéristiques, il faut signaler : i) l'emploi de la modélisation $3 \mathrm{D}$, contrairement au logiciel précédent; ii) l'animation

${ }^{26}$ Voir Fontanille, 2008. 
des marqueurs de poids indiquant le transfert du poids d'une jambe à l'autre; iii) le changement en cours d'œuvre du système de référenciation (à savoir de repérage cognitif et spatial dans l'espace de l'écran), qui permet à l'utilisateur de situer constamment sa position dans l'espace fictif des danseurs sur l'écran; iv) un système de vibration de l'appareil qui suit le rythme de la musique; et v) tout particulièrement, un système de captation du mouvement à la base de l'élaboration des silhouettes animées ainsi que des leurs mouvements. Comme dans le cas de Bailemos Tango, on retrouve ici une figurativisation ${ }^{27}$ multiple de la présentation des séquences à apprendre, par des silhouettes animées et par des diagrammes également animés (voir la figure Io). En revanche, le spectre des figures proposées est limité à un nombre exigu d'enchaînements considérés basiques que l'utilisateur peut sélectionner à tout moment. En effet, à la différence de Bailemos Tango, l'absence de numérotation dans la présentation des figures et en même temps l'absence d'une thématique à même de les profiler en les inscrivant dans un plus vaste cadre perceptif et culturel permettent d'un côté une incorporation et une répétition gestuelle dont la constitution est davantage déléguée à l'utilisateur, mais, d'un autre côté, détachent le corps-modèle du support de sa propre strate mémorielle (à la fois gestuelle et culturelle). Les corps tridimensionnels des danseurs reconstruits à partir du système de captation $d u$ mouvement se présentent comme des corps quelconques et en ceci anonymes, qui rendent la reprise gestuelle disponible à une singularisation opérée par chaque corps. Cet aspect se manifeste non seulement à travers la constitution figurative des corps des danseurs, mais concerne également l'intégration de l'utilisateur dans l'espace de l'écran et la navigation à l'intérieur de celui-ci. En effet, l'application ne peut pas aspirer à un statut «environnemental » ou «atmosphérique », non seulement parce qu'elle est intégrée dans une prothèse, mais aussi parce qu'elle demeure un support d'un point de vue modal. En d'autres termes, bien que la responsabilité de l'incorporation et de la reprise gestuelle soit davantage déléguée à l'utilisateur, le dispositif garde néanmoins une factitivitée ${ }^{28}$ à lui propre, c'est-à-dire des déclinaisons établies du pouvoir-faire et du devoir-faire du sujet utilisateur qui restent l'apanage du dispositif. C'est la raison pour laquelle l'application cherche à constituer un véritable espace diagrammatique, un espace modal de relations fictives, qui puisse englober le corps de l'apprenti. Dès lors, si l'on suit un parcours qui va de

${ }^{27}$ Lorsque l'on parle de figurativisation, on entend tout processus de prise de forme et de reconnaissance dans une scène pratique ou fictionnelle. Ici, les silhouettes dessinent des corps génériques et pourtant reconnaissables en termes de répartition des rôles dans l'interaction.

${ }_{28}$ Par factivité l'on entend des possibilités d'action inscrites dans l'objet même. Il ne s'agit pas d'affordance, mais plutôt des possibilités de manipulation du faire des sujets. 
l'hétéronomie à l'autonomie dans la gestion du geste, il nous semble que l'application iTango! instaure une autonomisation guidée du geste à répéter.

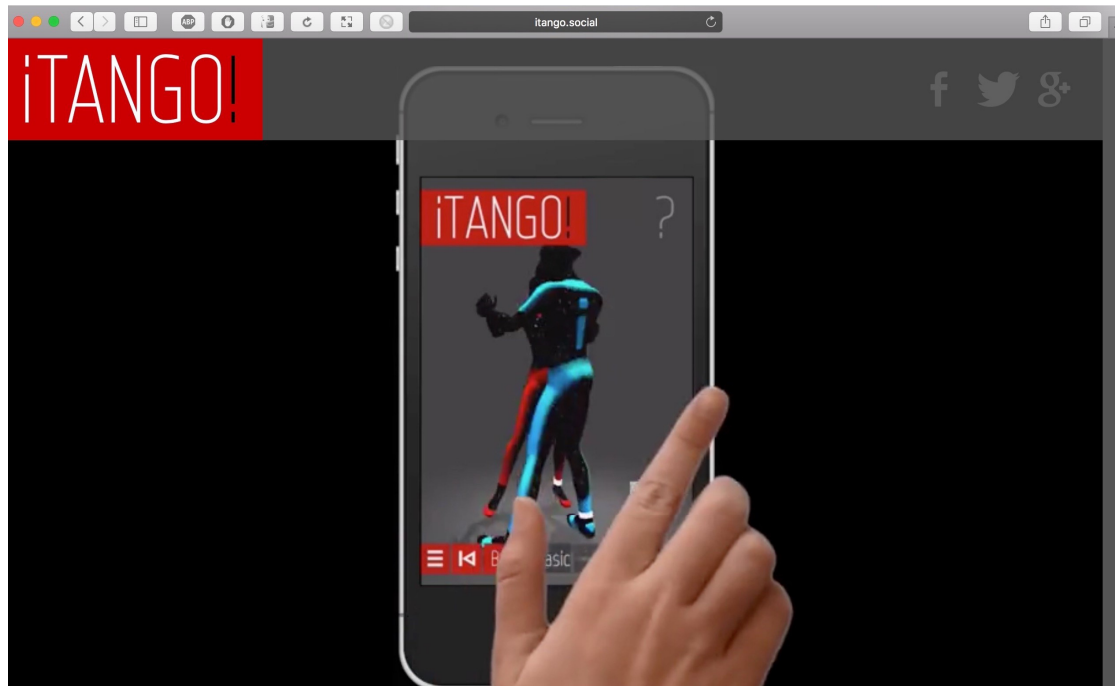

Fig. 9. «Interface iTango!», capture d'écran du site iTango!, 20I7, http://itango.social/ (consultation le 8 mai 2017).

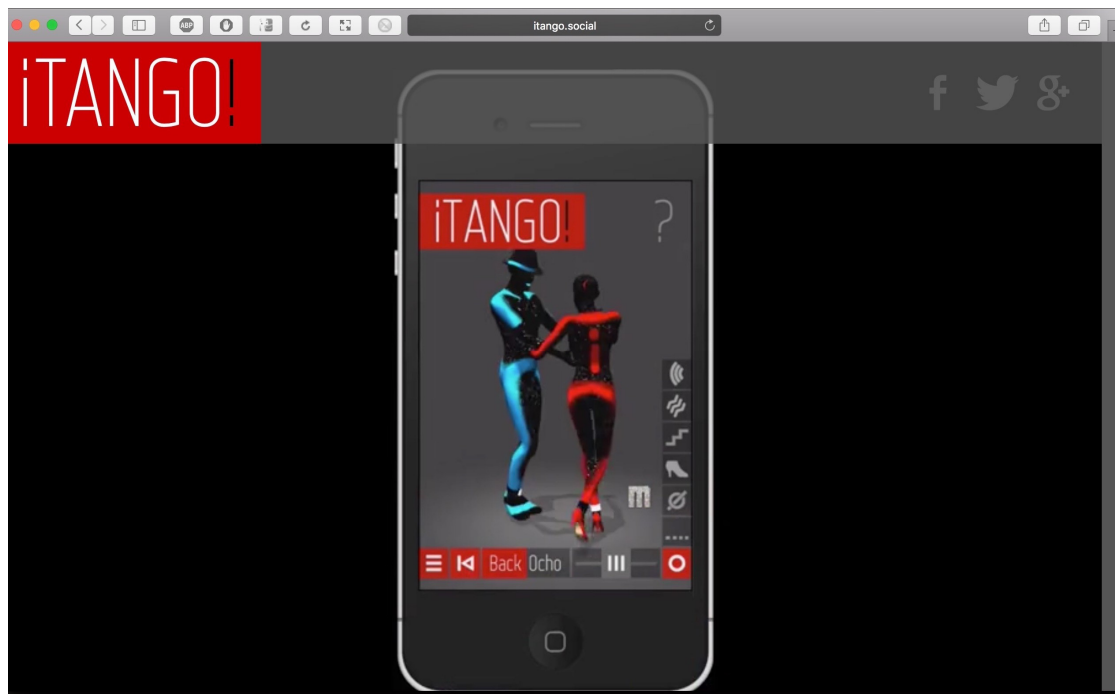

Fig. Io. «Mode silhouette », capture d'écran du site iTango!, 20I7, http://itango.social/ (consultation le 8 mai 20I7). 


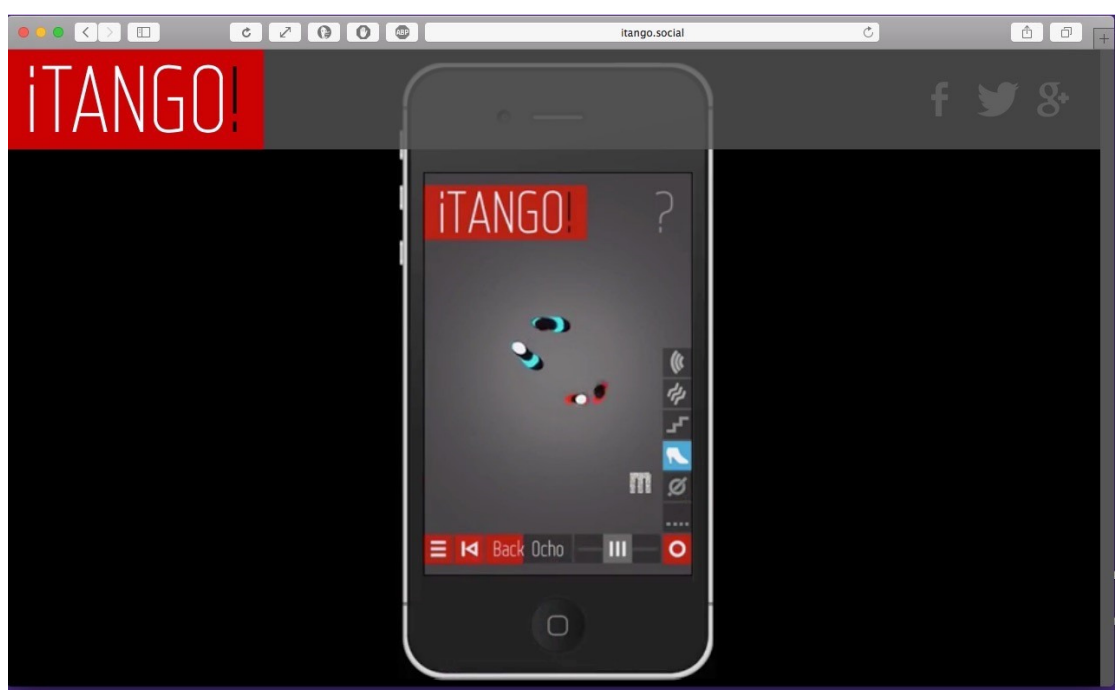

Fig. II. «Mode diagramme pieds», capture d'écran du site iTango!, 20I7, http://itango.social/ (consultation le 8 mai 2017).

\section{TANGO VIRUS}

L'installation Tango Virus (2005) fait partie d'une exploration plus vaste de la part du groupe argentin Proyecto Biopus ${ }^{29}$, formé d'une équipe d'ingénieurs, musiciens et artistes multimédias, qui travaille sur les différentes formes et technologies d'interaction et d'interactivité entre le public et les ouvres. Les chercheurs opèrent une manipulation d'algorithmes génétiques afin de créer un véritable milieu d'interaction - des boucles d'actions/réactions - entre l'environnement technologique, la musique et les danseurs. En particulier, comme le souligne la philosophe et sémioticienne Natalia Matewecki :

Les algorithmes évolutifs permettent de créer des œuvres moyennant des chaînes de bits qui aident à trouver la solution à un problème issu de processus biologiques-évolutifs tels le surpeuplement, la variabilité et l'héritage. D’autre part, il existe des algorithmes génétiques évolutifs qui s’inspirent eux aussi de l'évolution biologique mais qui travaillent avec des enchaînements d'arborescences afin de trouver un programme capable de résoudre un

29 Voir le site Internet du Proyecto Biopus : www.biopus.com.ar/; et plus spécifiquement la page consacrée à l'installation Tango Virus (http://www.estudiobiopus.com.ar /estudio/tango_virus.html), et le site Internet du studio Biopus: Estudio Biopus, 2016, http://www.estudiobiopus.com.ar/estudio/ (consultation le 8 mai 20I7). 
problème. Les algorithmes génétiques se fondent sur la génétique moléculaire en ceci qu'ils font évoluer une population d'individus à travers des actions telles la mutation ou la recombinaison génétique. Cette deuxième classe d'algorithmes est celle employée dans Tango Virus ${ }^{30}$.

L'installation crée un véritable environnement de médiations à travers l'appareillage technologique prévoyant une multiplication des supports, de l'espace du bal. En effet, comme le montrent les figures de I2 à I4, le système d'éclairage identifie la zone de danse, flanquée de haut-parleurs et surplombée d'une caméra. À son tour, cet espace est démultiplié par un espace «miroir » constitué par deux écrans qui diffusent respectivement l'évolution virale du thème musical (écran de gauche) et les réponses générées par le traitement en temps réel des variations sonores engendrées par le virus de la part des danseurs et de leurs mouvements (écran de droite).

Plus précisément, dans une première phase, la danse improvisée du couple est captée et enregistrée par la caméra qui transmet à l'un de deux ordinateurs les données concernant, entre autres, la position et la vitesse du mouvement. Ces informations, une fois numérisées, constituent un pattern visuel qui, à son tour, génère un virus qui « attaque » le thème musical (il s'agit d'un morceau d'Astor Piazzolla). La trace corporelle du mouvement, convertie en virus, est projetée sur l'un des écrans, en même temps que son évolution en temps réel et ses interactions avec le couple dansant (voir les figures is et I6).

Dès lors, les effets - certes manipulés - des gestes effectués sont restitués aux danseurs (et au public) à la fois visuellement et musicalement, dans la mesure où l'évolution du virus dépendra des ajustements corporels mis en place par les danseurs par rapport à la musique. Ici se joue un véritable dédoublement de l'assomption du geste, de l'improvisation et de sa propre reprise : les danseurs doivent en effet, d'une part, essayer de garder la synchronisation et un ajustement interprétatif avec la musique « infectée », tandis que, d'autre part, ils doivent éviter de répéter les

30 « los algoritmos evolutivos permitan crear obras digitales mediante el trabajo con cadenas de bits que posibilitan hallar la solución a un problema basado en procesos biológicos evolutivos como la superpoblación, la variabilidad y la herencia. Por otra parte, existen los algoritmos genéticos que también se inspiran en la evolución biológica pero que trabajan con cadenas de árboles para encontrar un programa que resuelva el problema. Los algoritmos genéticos se basan en la genética molecular en tanto hacen evolucionar una población de individuos mediante acciones como la mutación y la recombinación genética. Esta segunda clase de algoritmos es utilizada en Tango virus », Natalia Matewecki, « El discurso de la biología en el arte argentino contemporáneo », Ensayos. Historia y Teoría del Arte, n ${ }^{\circ}$, 2008, Universidad Nacional de Colombia, p. 38-39 (notre traduction). 


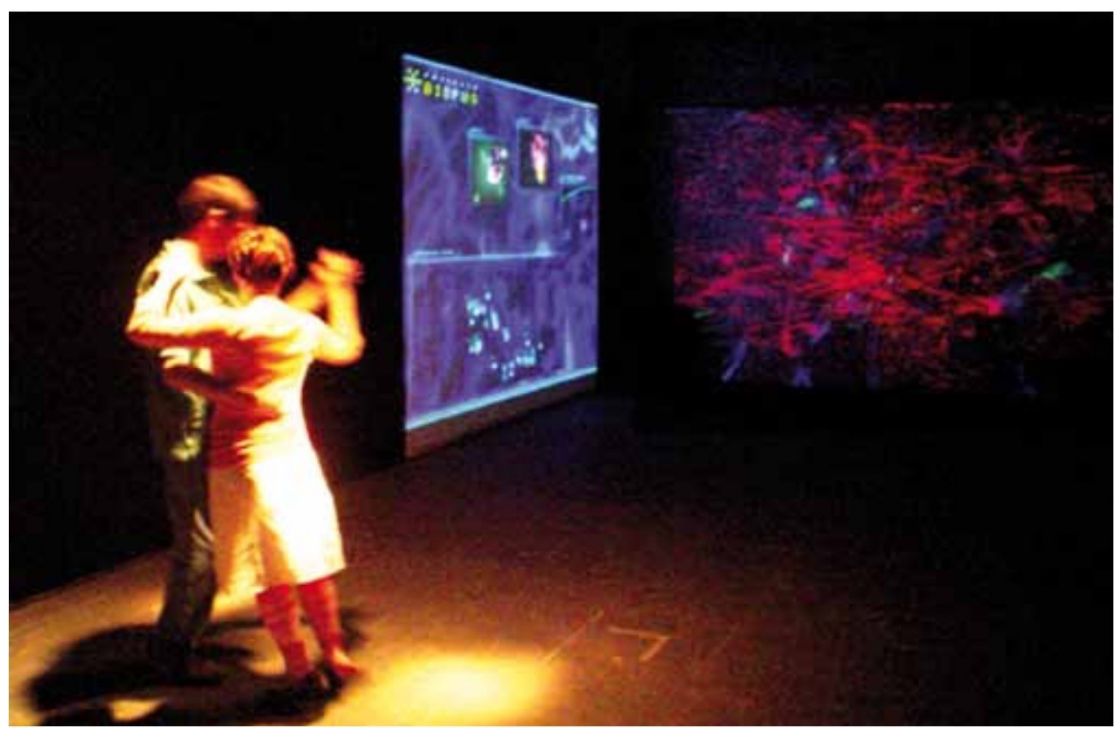

Fig. I2. Proyecto Biopus, Tango virus, installation, Espacio Fundación Telefónica, Buenos Aires, 2005.

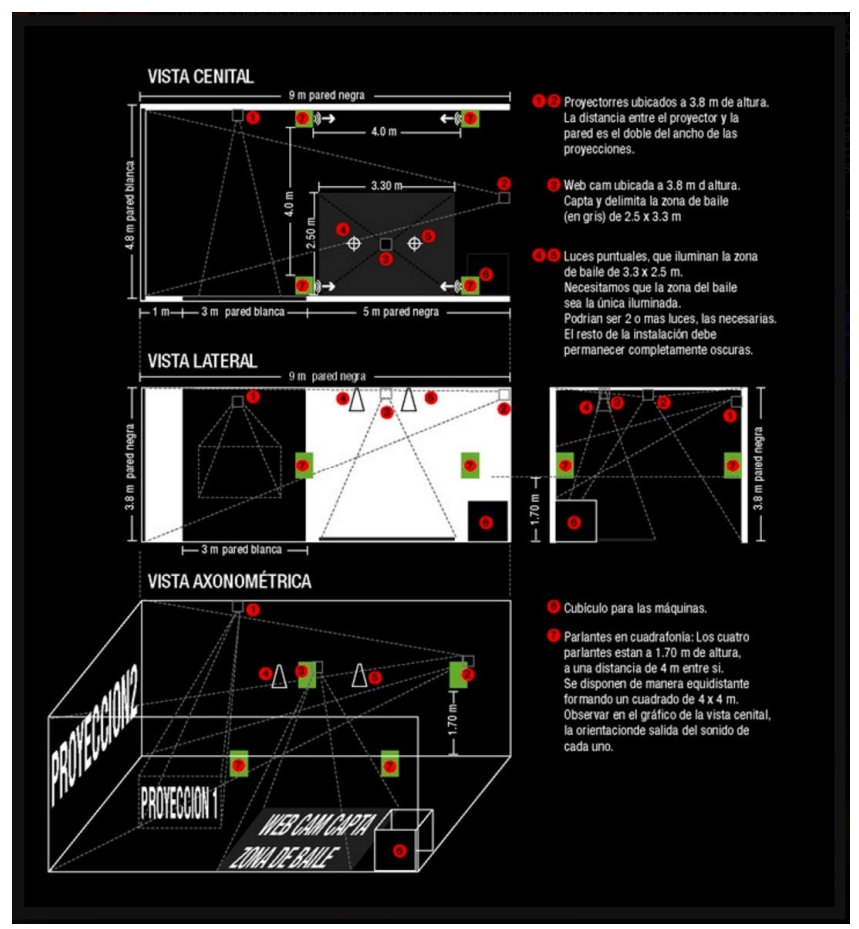

Fig. 13. Proyecto Biopus, Tango virus, installation, Espacio Fundación Telefónica, Buenos Aires, 2005, planimétrie. 


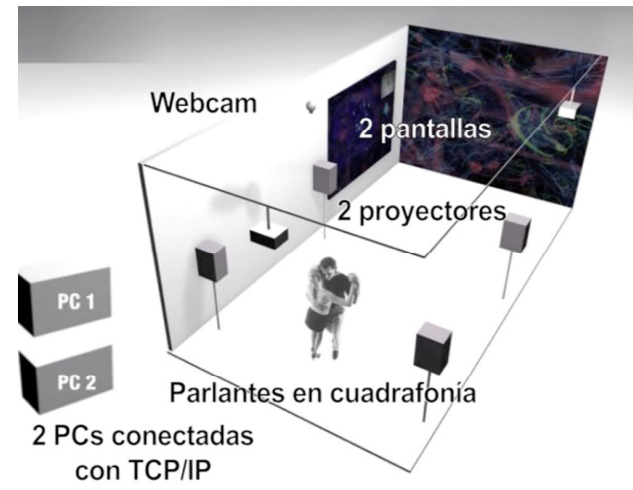

Fig. I4. Proyecto Biopus, Tango virus, installation, Espacio Fundación Telefónica, Buenos Aires, 2005, équipement technologique.

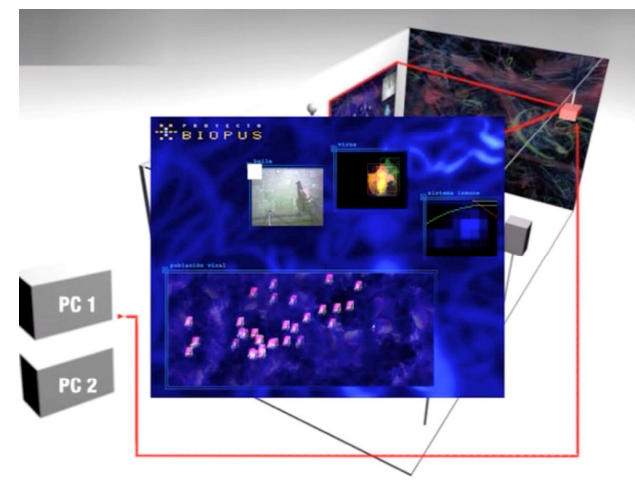

Fig. I5. Proyecto Biopus, Tango virus, installation, Espacio Fundación Telefónica, Buenos Aires, 2005, écran pattern visuel.

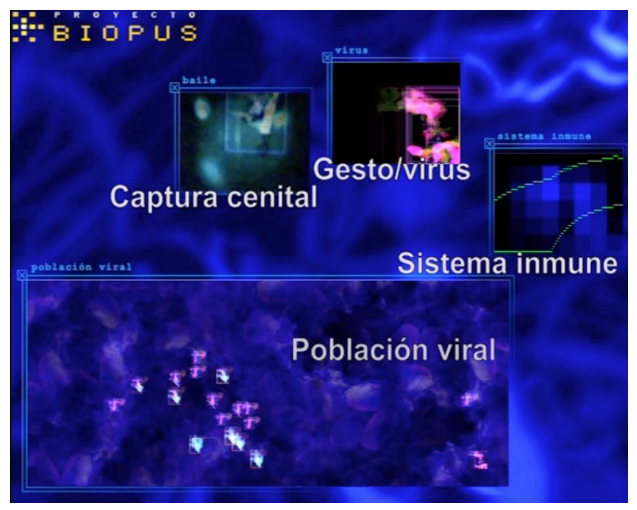

Fig. 16. Proyecto Biopus, Tango virus, installation, Espacio Fundación Telefónica, Buenos Aires, 2005, interactions du système. 
patterns visuels à l'origine de la contamination virale, bien qu'ils ne puissent pas savoir exactement, en vertu du caractère improvisationnel de l'interaction, quels patterns sont inédits et quels patterns ont déjà été exécutés. En fonction du type particulier d'enchaînement improvisé - qui sera littéralement couplé avec le système musical-viral -, qui est déployé, deux développements principaux sont possibles: d'un côté, le rétablissement d'un état d'immunisation à l'égard du virus ou bien, de l'autre côté, la mort du thème musical. Dès lors, le sort non seulement de l'interaction gestuelle entre les partenaires, mais aussi celui de toute la pratique, est à la charge des danseurs. L'autonomisation, ainsi que la possibilité de contrôle rétroactif et instantané des répétitions gestuelles improvisées sont pourvues par la médiatisation de l'environnement de la pratique, par laquelle l'appareillage technologique n'est pas qu'un simple support, mais devient un acteur métabolique à part entière qui oblige les danseurs à rectifier instantanément leur propre improvisation/reprise gestuelle.

\section{INTERACTIVE TANGO MILONGA}

Le projet Interactive Tango Milonga radicalise, quant à lui, la constitution d'un milieu médiatisé qui affiche la réflexivité du geste dansant, sur lequel les corps peuvent rétroagir de différentes manières. Dans ce cas, la dynamique d'improvisation/reprise se traduit par une véritable création et non pas seulement par une réponse plus ou moins adaptative comme dans le cas de Tango Virus. En effet, comme l'affirme la chercheuse et danseuse Courtney Brown, porteuse du projet, « the aim of Interactive Tango Milonga, is to foster a sense of individual agency over the music, allowing a measure of musical intentionally to each dancer ${ }^{31} \gg$. L'expérience se base sur les possibilités offertes par l'exploitation des systèmes de captation du mouvement. À cet égard, il faut préciser que plusieurs types de capteurs sont testés - des capteurs optiques aux capteurs portables — sur et avec les danseurs. Les figures I7 et I8 montrent les capteurs portables, choisis pour des raisons techniques de préservation du signal, que les danseurs portent aux chevilles ainsi qu'autour du torse, afin de restituer des données différentes du mouvement. L'objectif de l'expérience, réalisée à l'aide de caméras et de logiciels de transformation

${ }^{31}$ Courtney Brown et Garth Paine, « Towards an interactive Tango Milonga », Actes de la $4 I^{e}$ International Computer Music Conference: «Looking Back, Looking Forward», 25 septembre- $\mathrm{I}^{\mathrm{er}}$ octobre 20I5, Denton, États-Unis, San Francisco, The International Computer Music Association, p. III. Pour un aperçu visuel de l'expérience, consulter le site internet du projet: Courtney Brown, Interactive Tango Milonga: An interactive dance system for Argentine social tango dance, 2015, www.interactivetango.com/ (consultation le 8 mai 2017). 

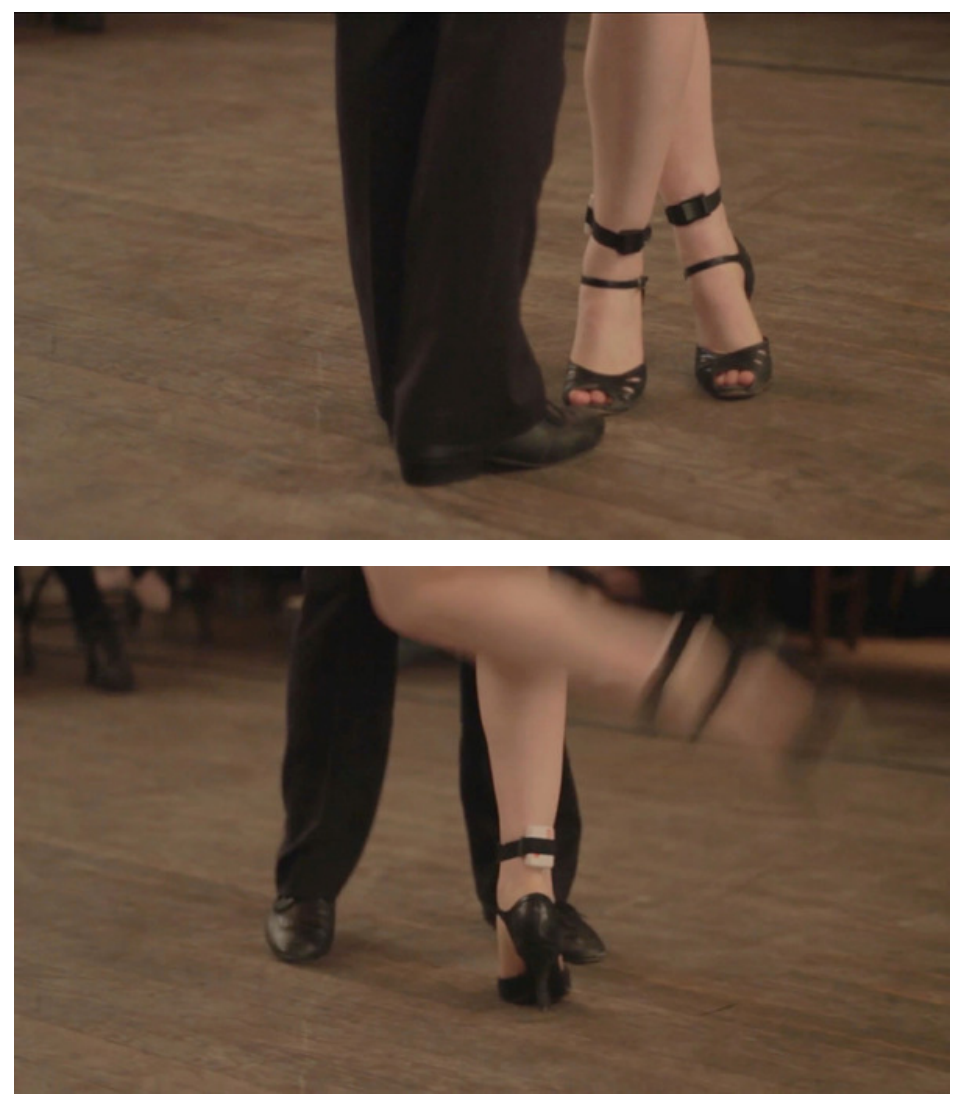

Fig. 17-I8. Interactive Tango Milonga, capteurs (recto), avec l'aimable autorisation de Courtney Brown.

et de traitement du signal ainsi que de composition musicale, consiste en la génération de boucles sonores qui réagissent en fonction des figures effectuées par les danseurs et sur lesquelles ils peuvent rétroagir en réajustant leur propre danse. Autrement dit, ce sont les gestes des danseurs qui, une fois numérisés, sont implémentés dans une base rythmique donnée pour créer un morceau de tango ou de milonga. Comme on peut le constater, le degré d'assomption de l'improvisation/reprise est maximal, dans la mesure où tout pattern gestuel, qu'il soit répété de la « même » manière ou non, devient un outil de recréation continue. Autrement dit, ce qui fait l'objet d'une réflexivité maximale est précisément la capacité des danseurs de se coordonner et d'intégrer un morceau réellement inédit. Actuellement, l'expérience n'a été conduite que sur un seul couple, bien que le projet vise à une extension à plusieurs couples et notamment à la réalisation d'une expérience collective dans l'espace de la milonga. 
Néanmoins, la multiplication des acteurs ne fait qu'amplifier les problèmes rencontrés avec le couple test par rapport au choix du système de captation ainsi que de la technologie de transmission du signal. En effet, les facteurs qui doivent être pris en compte sont nombreux et différents les uns des autres, allant de la nécessité d'encombrer le moins possible les danseurs à la distinction des parties du corps de chaque danseur. Finalement, la médiatisation de l'environnement de la pratique du bal, obtenue cette fois-ci par une incorporation d'une partie de l'équipement technique, semble constituer une solution relativement efficace afin de restituer la dynamique entre improvisation et reprise à l'œuvre dans le tango.

\section{CONCLUSIONS. L'ANARCHIVE EN TANT QUE REENACTEMENT MÉDIAL}

Bien qu'il soit impossible de déterminer de véritables conclusions, vu le caractère in fieri des dispositifs, des expérimentations et des environnements médiatiques examinés ici - susceptibles de mises à jour technologiques, de changements dans leur design, de variations spatiales et d'usage - , essayons tout de même de fournir des éléments de réponse qui peuvent guider des diffractions médiales à venir. À ce propos, une déclinaison différente de la notion d'interaction ainsi qu'une conception différente du médium constituent à notre avis la clef de voûte pour comprendre le reenactement médial du tango. En d'autres termes, pour que l'on puisse parler d'un reenactement médial de l'interaction dansante du tango, il faut d'un côté que la médiatisation ne soit pas réduite à l'interaction avec un support (objet, prothèse ou substitut d'une partition) et que, d'un autre côté, on prenne au sérieux l'idée d'une interaction entre des corps - voire des êtres possédant des matières expressives différentes. Dans ce cadre, les deux derniers exemples semblent suivre cette piste, dans la mesure où non seulement les outils technologiques médiatisent les corps des danseurs, mais ce sont aussi ces derniers qui « corporalisent » les premiers dans un environnement décidément hybride. L'accent porté sur l'hybridité de cet environnement oblige à repenser le nombre d'acteurs ainsi que leurs rôles respectifs dans le cadre de la pratique du bal. Dans ce sens, la hiérarchie implicite suivant laquelle les corps reenacteraient eux-mêmes d'abord pour être soumis ensuite à un reenactement médial est mise en crise par l'idée même d'un environnement médiatique. Autrement dit, celui-ci permettrait, d'un côté, de dépotentialiser l'idée d'une mémoire exclusivement corporelle — bien qu'elle soit partagée - et, de l'autre côté, de dépotentialiser la tentation à l'archive 
(traditionnellement conçue), qui affecte les outils numériques à l'égard du traitement du signal et de l'information.

C'est dans cette acception que l'on pourrait concevoir les environnements médiatiques comme des formes d'anarchive, suivant les traits programmatiques identifiés par l'équipe de recherche montréalaise du SenseLab ${ }^{32}$, parmi lesquels figurent la non-objectualité de l'anarchive, la nature de phénomène multi-plateforme, ainsi que sa nature foncièrement processuelle. Bien que nous n'ayons pas ici le temps d'approfondir toutes les nuances d'une notion elle-même à l'état germinatif, soulignons, avec Brian Massumi, que « the anarchive is not documentation of a past activity. Rather, it is a feed-forward mechanism for lines of creative process, under continuing variation. The anarchive needs documentation - the archive - from which to depart and through which to pass ${ }^{33}$. » En d'autres termes, l'anarchive ainsi conçue semble mettre en crise précisément le rapport à la trace qui, suivant Lepecki, révèle le caractère de dispositif de toute archive, « by dictating what deserves a place in it, and what should be excluded from it, by determining what is to be properly filed and what is (purposefully or inadvertently) to be "misplaced" in it ${ }^{34} \gg$. Dans cette perspective, la trajectoire descriptive suivie montre, de manière comparée, une progression d'outils et média se situant idéalement du côté de l'archive (les deux premiers cas d'étude) vers des environnements qui semblent se placer davantage du côté de l'anarchive, en ceci qu'ils comportent une dimension véritablement événementielle et prennent davantage en compte les réponses des acteurs. Finalement, les environnements médiatiques ainsi conçus, qui seuls peuvent actuellement représenter des formes de reenactement médial, pourraient dans un futur prochain s'intégrer à et intégrer davantage les composants praxiques et normatifs de la pratique du bal et d'apprentissage, en prenant en compte non seulement la scansion rythmique d'un morceau de musique, mais aussi, par exemple, la scansion temporelle globale de la pratique. En conclusion, cela pourrait ouvrir une voie pour l'incorporation du seul facteur qui résiste encore à tout support, à toute inscription, c'est-à-dire le caractère collectif de la pratique.

32 Voir notamment le blogue de SenseLab, Wordpress, http://senselab.ca/wp2 /immediations/anarchiving/anarchive-concise-definition/ (consultation le 8 mai 20I7).

${ }_{33}$ Brian Massumi, « Working Principles », dans Andrew Murphie (dir.), The Go-To How To Book of Anarchiving, Montréal, SenseLab, 2016, p. 6.

34 Lepecki, 2oro, p. 30. 


\title{
Le tango argentin entre apprentissage et improvisation. Quel média pour quel reenactment ?
}

\author{
Valeria De luca, Université de limoges
}

\section{RESUME}

Inscrit en 2009 sur la liste du patrimoine culturel immatériel de l'humanité de l'UNESCO, le tango argentin est transmis par sa propre pratique caractérisée par l'improvisation de la milonga (le bal).Avec la multiplication de traces audiovisuelles diverses - des vidéos de démonstration aux systèmes de motion capture, en passant par des sites web et des applications pour l'apprentissage de la danse -, il s'agit de comprendre les relations entre l'improvisation comme pratique fondatrice du tango et ses diffractions médiales, ainsi que le statut de ces diffractions. Quels aspects $\mathrm{du}$ mouvement et de la pratique restituent-elles ? Sont-elles elles-mêmes un reenactement médial se superposant à celui des corps des sujets ?

\section{ABSTRACT}

Inscribed in 2009 on UNESCO's "Representative List of the Intangible Cultural Heritage of Humanity," the Argentinian and Uruguayan Tango is a practice transmitted through improvisation within the social framework of the milonga (the place where the tango is danced). Given the proliferation of audiovisual traces-from demonstration videos to motion capture systems, websites and learning applications-it is worth understanding the relationship between improvisation as a foundational practice of tango, and its various media representations, as well as the status of these forms in relation to the practice. What aspects of movement and dance do they reveal? Do they constitute a medial re-enactment imposed, in turn, on the dancing bodies of the subjects?

\section{NOTE BIOGRAPHIQUE}

Valeria De luca est docteure en Sciences du langage-Sémiotique à l'Université de Limoges. Ses travaux portent sur la danse et tout particulièrement sur l'univers et la pratique du tango argentin, ainsi que sur les relations entre geste, culture et identité. Parmi ses publications principales : «Valeur, sens et énonciation. Ce que Dewey fait à la sémiotique » (Versus, $\left.\mathrm{n}^{\circ} \mathrm{I23}, 2016\right)$; «La matière et la technique comme dispositifs de médiation. Le cas des Cartes-tapisseries d'Alighiero Boetti » (Actes AFS, 2016); «Le figural entre imagination et perception » (Metodo. International Studies in Phenomenology and Philosophy, vol. 3, n I, 2015). 